unique challenge in inpatient psychiatry settings both in terms of diagnosis and treatment. This is perhaps greater still in forensic settings due to the increased risk of violence and aggression. This audit aimed to firstly assess the consistency of local practice to national guidance from Public Health England. Secondly, it aimed to describe the clinical management of suspected and confirmed cases of COVID-19 in this high security forensic hospital and how readily broad, national guidance can be implemented in this unique setting. We present an audit with three cycles, one from each wave of COVID-19 in England during 2020.

Method. This was a retrospective audit in a high secure forensic psychiatry hospital in the United Kingdom, into the investigation and management of suspected and confirmed cases of COVID-19 compared to national guidelines from Public Health England. It includes three cycles, one undertaken in each national wave of COVID-19 in England in 2020.

Result. Ten patients have been included in cycle 1, 12 in cycle 2 and 21 in cycle 3 as those where COVID-19 was a considered diagnosis. SARS-CoV-2 was detected in one patient in cycle 1 and 12 patients in cycle 3. All patients recovered, most of whom remained on-site with supportive care in self-isolation on a dedicated ward for positive cases. Three patients required additional treated with oral antibiotics and dexamethasone, one of whom required admission to the local general medical hospital for continuous supplemental oxygen.

Conclusion. This is the first study to describe the management of the novel COVID-19 pandemic in a high security forensic psychiatry hospital and how readily national guidance can be implemented in this unique setting. Hospital practice at identifying suspected cases and the management of confirmed cases of COVID-19 was shown to be consistent with national guidance. It also allowed for clinicians to exercise their judgement about testing for atypical cases and for repeat testing where appropriate.

Audit examining memantine initiation in dementia patients in an older adult service in the north west

Irina Casapu ${ }^{1 *}$, Ste Dickinson ${ }^{2}$, Chirag Shroff ${ }^{3}$, Sofia Almeida ${ }^{4}$ and Kieran McSharry

${ }^{1}$ CT3, Liaison Psychiatry, Aintree Hospital; ${ }^{2}$ F3, A and E, Royal Liverpool University Hospital; ${ }^{3}$ CT2, Burlington House CAMHS, Alderhey NHS Trust; ${ }^{4}$ CT3, Liaison Psychiatry, Countess of Chester Hospital and ${ }^{5}$ ST5 Dual Old Age and General Adult Trainee, Merseycare NHS

${ }^{*}$ Corresponding author.

doi: 10.1192/bjo.2021.225

Aims. Dementia is a progressive condition inflicting significant costs for health and social care services. In December 2017, there were 456,739 people on GP registers with a formal diagnosis of dementia. Making the right choice of anti-dementia medication with essential monitoring is one important aspect of care. Thus, the aim of this audit was to identify if current practice at Mossley Hill inpatients and outpatients service for older adults in Liverpool, was in accordance with the NICE Guideline NG97 (Dementia: assessment, management and support for people living with dementia and their carers). Additionally, we aimed to evaluate whether Memantine was commenced according to $\mathrm{BNF} / \mathrm{SPC}$ recommendations about e-GFR and whether this was documented on patient records, as well as to highlight areas of improvement.

Method. An audit was carried out for all patients for whom Memantine was initiated, between June and August 2019. Sixty-nine patients were identified through trust Pharmacy records. Data were collected retrospectively, reviewing local electronic records (ePEX, RIO) and GP referrals. This included age, sex, diagnosis, indication for starting Memantine, decision context, prescriber, documentation of renal function status and communication of decision to the GP. Findings were compared to NICE guidance NG97 and presented at the local audit meeting with a view to recommend strategies for improvement.

Result. Results indicated that most of the patients were female (64\%) with the most common diagnosis being Alzheimer's disease (75\%). Recurrent reasons for initiating Memantine were: contraindication for AChE treatment (25\%); illness progression on AChE (22\%); and severe dementia on initial presentation (23\%). Usually, the decision to start Memantine treatment was made in MDT or after prescriber clinical review. In $68 \%$ of the reviewed cases, renal function status was documented. Patients' GP was informed of medication change in $86 \%$ of cases.

Conclusion. To conclude, in the majority of cases Memantine initiation was in line with NICE guidance. However, documentation can be improved, so as to facilitate future audit. We recommended creating a checklist for prescribing Memantine that could be integrated within the electronic records system.

\section{Transition from Methylphenidate to Atomoxetine: reasons for switching and clinical outcome}

Fabrizia Cassar ${ }^{\star}$, Giovanni Grech, Bertha Grech and Joseph Cassar

Mount Carmel Hospital

${ }^{\star}$ Corresponding author.

doi: 10.1192/bjo.2021.226

Aims. Attention Deficit Hyperactivity Disorder (ADHD) is a behavior disorder originating in childhood comprising of a constellation of features including inattention, impulsivity, and hyperactivity. The National Institute of Clinical Excellence (NICE) Guidelines 2018 recommends methylphenidate as a first line pharmacological agent for treatment of children aged 5 years and over with ADHD. Lisdexamfetamine, dexamfetamine and atomoxetine are recommended in this order if methylphenidate is not tolerated or if symptoms did not respond to separate 6-week trials. Our aim was to, assess the transition of methylphenidate to atomoxetine, the reasons for switching and its clinical outcome in order to make recommendations to current practice regarding treatment of ADHD.

Method. The study examined a total of 53 children between 0-16 years of age who were being treated for ADHD with atomoxetine at CYPS till September 2018. Data was collected from patients' files retrospectively by using a proforma based on the NICE guidelines 2018 ADHD: diagnosis and management.

Result. Out of 53 patients' on atomoxetine in September 2018, 49 were included in the study. Results recorded side-effects as the main reason for switching from methylphenidate to atomoxetine. Unwanted side-effects were documented in $71.7 \%$ of patients of which $57.9 \%$ exhibited more than 1 side-effect with the two commonest side-effects documented being weight loss and decreased appetite. The audit highlighted the fact that the correct dose of atomoxetine was only administered in $17.2 \%$ of children with $56.9 \%$ of patient's being given a higher dose than recommended. Initial weight was not documented in $19 \%$ and hence, ideal dose could not be calculated. Overall, atomoxetine was shown to be an effective treatment. Out of the 40 patients documented to have hyperactivity this symptom was decreased in $82.5 \%$ whilst $82.9 \%$ were shown to have increased concentration. 35 patients had documented impulsivity and this was decreased in $62.9 \%$ of 
cases. 11 patients had documented anxiety with $72.7 \%$ being treated effectively with atomoxetine. $31 \%$ of patients' had documented side-effects with $16 \%$ of these being tics. $20 \%$ of patient's required augmentation.

Conclusion. The results indicate that the majority of doctors at CYPS in Malta adhered to the NICE guidelines 2018 and atomoxetine was proven to be efficacious as a second line drug in the treatment of ADHD. However, better adherence to NICE guidelines is required when it comes to the calculation of appropriate dosage. Our prediction is had dose recommendations according to weight been adhered to there may have been less side-effects documented.

\section{Audit: lithium monitoring for psychiatric inpatients and community patients during the initiation phase}

Damodar Chari, Mohammed Abbas and Kajal Dhesi

Leicestershire Partnership Trust

${ }^{\star}$ Corresponding author.

doi: 10.1192/bjo.2021.227

Aims. Measure compliance with standards requiring baseline work up before Lithium therapy is commenced and subsequent Lithium level monitoring during the initiation phase

Method. All inpatients and outpatients who were started on Lithium between 2018 and 2019 within the Leicestershire Partnership NHS trust. Case notes were of patients 128 were retrieved from the electronic system and an audit proforma was completed to ascertain adherence to auditing standards as per $\mathrm{BNF}$ and trust guidelines. Parameters monitored were full blood count $(\mathrm{FBC})$, renal functions test including serum electrolytes, thyroid function test, and BMI before commencing Lithium, and serum Lithium periodically after. ECG was needed for those patients with cardiovascular illness. Data were systematically compiled and analyzed descriptively using Microsoft Excel

Result. A total of 128 patients were included in the study. 111 (86.71\%) had FBC, 118 (92.19\%) had renal function test and electrolytes, 114 (89.06\%) had thyroid function test while 99 (77.34\%) had their BMI/weight recorded before initiating Lithium. 26 out of 36 patients with cardiovascular disorder had their ECG recorded. After Lithium was commenced, 108 (84.37\%) had serum Lithium tested a week later, while only 89 (69.53\%) had lithium monitored weekly. Trust guidelines recommend weekly monitoring for up to 4 weeks after a stable dose was reached. This was monitored in only 16 out of 128 patients.

Conclusion. Most of the patients had blood test done before being commenced on Lithium. However it was observed that serum Lithium was not adequately monitored at regular intervals after dose escalations. These finding indicate that there has to be greater awareness of the trust and BNF guidelines with regards to Lithium monitoring.

\section{Hyponatraemia monitoring in those prescribed} antidepressants - an audit from an inpatient older adult ward

Lorena Chavez*, Jake Scott, Richard Hoile, Jane McNulty and Louisa Marchant-Rutherford

Sussex Partnership NHS Foundation Trust

${ }^{\star}$ Corresponding author.

doi: 10.1192/bjo.2021.228

Aims. To assess follow-up of sodium levels for in-patients prescribed antidepressants in practice compare to the standard of 3 monthly sodium levels for all patients who are prescribed antidepressants and at risk of hyponatraemia

Method. A list of the 20 most recently discharged patients from Meridian Ward, an older-adult functional inpatient ward, was prepared by the team administrator on 6th May 2020.

We audited the entire duration of our patient's stay on Meridian Ward (we did not include periods of their admission when they were on other wards) using the electronic notes system, Carenotes.

We also checked the electronic biochemistry results system, ICE, for sodium results, and the discharge summary for mentions of fluid restriction, medications and handover to GP of sodiumchecking. We also checked scanned drug charts to see if they were on antidepressants and other implicated drugs.

For people with episodes of hyponatraemia, in order to retrieve further info we looked at discharge summary and searched the activity notes for the following terms

"Hyponat"

"sodium"

"fluid restrict"

"Low na"

We regarded the following conditions as risk factors for hyponatraemia:

cardiac

malignancy

respiratory

hypothyroid

renal

hepatic

stroke

We regarded following medications as risk factors:

opioids

diuretics

carbamazepine

theophylline

antipsychotics

NSAIDs

PPIs

ACE-I

ARBs

amiodarone

domperidone

sulphonylureas

Result. 14 of the 20 patients were taking antidepressants. Of those: 13 were eligible for regular sodium monitoring due to risk factors 11 of these had 3-monthly sodium levels during admission For only 2 of these did we make a plan for the GP to continue to monitor the sodium level in community 3 had an episode of hyponatraemia implicated antidepressants: sertraline plus mirtazapine mirtazapine (very serious episode which caused seizure) sertraline for 2 of them an appropriate plan was made 1 without a plan - a mild hyponatraemia with nothing documented in the notes

Conclusion. During their admission to Meridian Ward, 85\% of patients taking antidepressants who had risk factors for hyponatraemia had three-monthly sodium levels in line with the trust guidance. However, only two patients (15\%) had a plan for further sodium levels in the discharge summary sent to the GP. This highlights a need for improved awareness of risk factors for hyponatraemia and, in particular, improved communication with general practitioners who are going to take over prescribing of antidepressant medications.

Recommendations

3 monthly $\mathrm{Na}$ levels for all patients with risk factors

i.e. on any antidepressant prescribed PLUS any one of: 